\title{
Reconstructing Shape from Motion Using Tactile Sensors*
}

\author{
Mark Moll \\ mmoll@cs.cmu.edu \\ Michael A. Erdmann \\ me@cs.cmu.edu \\ Department of Computer Science, Carnegie Mellon University \\ Pittsburgh, PA 15213
}

\begin{abstract}
We present a new method to reconstruct the shape of an unknown object using tactile sensors without requiring object immobilization. Instead, the robot manipulates the object without prehension. The robot infers the shape, motion and center of mass of the object based on the motion of the contact points as measured by tactile sensors. Our analysis is supported by simulation and experimental results.
\end{abstract}

\section{Introduction}

Robotic manipulation of objects of unknown shape and weight is very difficult. To manipulate an object reliably a robot typically requires precise information about the object's shape and mass properties. Humans, on the other hand, seem to have few problems with manipulating objects of unknown shape and weight. For example, Klatzky et al. (1985) showed that blindfolded human observers identified 100 common objects with over $96 \%$ accuracy, in only 1 to 2 seconds for most objects. So somehow during the manipulation of an unknown object the tactile sensors in the human hand give enough information to find the pose and shape of that object. At the same time some mass properties of the object are inferred to determine a good grasp. These observations are an important motivation for our research. In this paper we present a model that integrates manipulation and tactile sensing. We derive equations for the shape and mo- tion of an unknown object as a function of the motion of the manipulators and the sensor readings.

Figure 1 illustrates the basic idea. There are two palms that each have one rotational degree of freedom at the point where they connect, allowing the robot to change the angle between palm 1 and palm 2 and between the palms and the global frame. As the robot changes the palm angles it keeps track of the contact points through tactile elements on the palms.

In the next section we will give an overview of related work. In section 3 we derive the shape and motion of an unknown object as a function of palm motions and sensor values. In 4 we present simulation results for the same object that we used in our experiments. Some preliminary experimental results are presented in section 5. Finally, in section 6 we discuss briefly some directions we will explore in future work.

\section{Related Work}

Our research builds on many different areas in robotics. These areas can be roughly divided into four different categories: probing, nonprehensile manipulation, grasping, and tactile sensing. We can divide the related work in tactile sensing further into three subcategories: shape and pose recognition with tactile sensors, tactile exploration, and tactile sensor design. We now briefly discuss some of the research in these areas.

*This work was supported in part by the NSF under grants IIS-9820180 and IRI-9503648.

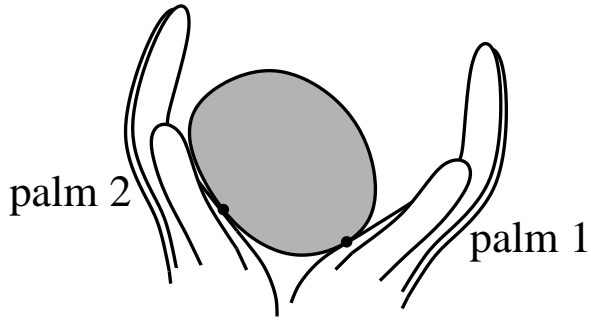

(a) Two palms



(b) Two fingers

Figure 1: Two possible arrangements of a smooth convex object resting on palms that are covered with tactile sensors. 


\subsection{Probing}

Detecting information about an object with sensors can be phrased in a purely geometric way. Sensing is then often called probing. One can define different kinds of probes that correspond to abstractions of sensor devices. For instance, a finger probe corresponds to a robotic finger moving along a line until it contacts an object (or misses the object). The probe outcome is then the point where the probe contacted the object. Cole and Yap (1987) showed that we can reconstruct a convex $n$-sided polygon using no more than $3 n$ finger probes. Furthermore, they showed that $3 n-1$ probes are necessary. Shortly after (Cole and Yap, 1987) Dobkin et al. (1986) investigated the complexity of determining the shape and pose of convex polytopes for a variety of different probes, and also probes with errors. Boissonnat and Yvinec (1992) extended the probe model of Cole and Yap: their probe outcome includes the normal at the contact point. With this probe model they show that at most $3 n-3$ probes are needed for simple non-convex polygons with no collinear edges.

$\mathrm{Li}$ (1988) gave algorithms that reconstruct convex polygons with $3 n+1$ line probes or with $3 n-2$ projection probes. Line probes slide a straight line in a particular direction over the plane until it hits the object. Projection probes consist of two line probes that move in opposite directions towards each other. Lindenbaum and Bruckstein (1994) gave an approximation algorithm for arbitrary planar convex shapes using line probes. In (Lindenbaum and Bruckstein, 1991) bounds were given on the number of parallel probes that are necessary to recover the shape of a planar polygon. With parallel probes, $k$ probes $(k>1)$ are performed at the same time. Skiena (1989) observed that the line probe can be generalized to a new kind of probe which is the dual of the finger probe, so that there is a one-to-one correspondence between algorithms that use finger probes and ones that use this generalized line probe.

Rao and Goldberg (1994) studied the problem of determining the shape of a convex polygon using diameter measurements from a parallel jaw gripper. They showed that it is possible to recognize a shape from a known (finite) set of shapes. Arkin et al. (1998) proved that finding a minimal length plan is $N P$-hard and give a polynomial-time approximation algorithm with a good performance guarantee. Akella and Mason (1998) showed how to orient and distinguish (sets of) polygonal parts using diameter measurements.

\subsection{Nonprehensile Manipulation}

The basic idea behind nonprehensile manipulation is that robots can manipulate objects even if the robots do not have full control over these objects. This idea was pioneered by Mason. In his Ph.D. thesis (Mason, 1982, 1985) nonprehensile manipulation takes the form of pushing an object in the plane to reduce uncertainty about the object's pose. Subsequent work by others showed how to use pushing to design fences for a conveyor belt system (Peshkin and Sanderson, 1988; Wiegley et al., 1996) and how to plan paths for pushing objects among obstacles (Lynch, 1997). Lynch et al. (1998) showed how to make a robotic manipulator perform a certain juggling motion with a suitable parameterization of the shape and motion of the manipulator. Much research on juggling balls has been done in Koditschek's research group (see e.g. (Rizzi and Koditschek, 1993) and (Whitcomb et al., 1993)).

Erdmann and Mason (1988) described sensorless manipulation within the formal framework of the pre-image methodology. In particular, Erdmann and Mason showed how to orient a planar object by a tray tilting device. In (Erdmann et al., 1993) the tray tilting idea is extended to polyhedra.

One of the first papers in palmar manipulation is (Salisbury, 1987). Paljug et al. (1994) investigated the problem of multi-arm manipulation. Erdmann (1998a) showed how to manipulate a known object with two palms. Zumel (1997) described a palmar system like the one shown in figure 1(b) (but without tactile sensors) that can orient known polygonal parts.

\subsection{Grasping}

The problem of grasping has been widely studied. This section will not try to give a complete overview of the results in this area, but instead just mention some of the work that is most important to our problem. In order to grasp an object we need to understand the kinematics of contact. Independently, Montana (1988) and Cai and Roth $(1986,1987)$ derived the relationship between the relative motion of two objects and the motion of their contact point. In (Montana, 1995) these results are extended to multi-fingered manipulation. Kao and Cutkosky (1992) presented a method for dextrous manipulation with sliding fingers.

Sudsang et al. (2000) looked at the problem of manipulating three-dimensional objects with a reconfigurable gripper. The gripper consists of two horizontal plates, of which the top one has a regular grid of actuated pins. Rao et al. (1994, 1995) show how to reorient a polyhedral object with pivoting grasps: the object is grasped with two hard finger contacts so that it pivots under gravity when lifted.

Trinkle and colleagues (Trinkle et al., 1993; Trinkle and Hunter, 1991; Trinkle and Paul, 1990; Trinkle et al., 1988) have investigated the problem of dexterous manipulation with frictionless contact. They analyzed the problem of lifting and manipulating an object with enveloping grasps. Yoshikawa et al. (1993) do not assume frictionless contacts and show how to regrasp an object using quasistatic slip motion. Nagata et al. (1993) describe a method of repeatedly regrasping an object to build up a model of its shape.

In (Teichmann and Mishra, 2000) an algorithm is presented that determines a good grasp for an unknown object using a parallel-jaw gripper equipped with some light beam sensors. This paper presents a tight integration of sensing and 
manipulation. Recently, Jia (2000) showed how to achieve an antipodal grasp of a curved planar object with two fingers.

\subsection{Shape and Pose Recognition}

The problem of shape and pose recognition can be stated as follows: suppose we have a known set of objects, how can we recognize one of the objects if it is in an unknown pose? For an infinite set of objects the problem is often phrased as: suppose we have a class of parametrized shapes, can we establish the parameters for an object from that class in an unknown pose? Schneiter and Sheridan (1990) developed a method for determining sensor paths to solve the first problem. In Siegel (1991) a different approach is taken: the pose of an object is determined by using an enveloping grasp.

Jia and Erdmann (1996) proposed a 'probing-style' solution: they determined possible poses for polygons from a finite set of possible poses. One method determined the pose by bounding the polygon by supporting lines. The second method they proposed is to sense by point sampling. They proved that solving this problem is $N P$-complete and present a polynomial time approximation algorithm.

Keren et al. (1998) proposed a method for recognizing three-dimensional objects using curve invariants. Jia and Erdmann (1999) investigated the problem of determining not only the pose, but also the motion of a known object. The pose and motion of the object are inferred simultaneously while a robotic finger pushes the object.

\subsection{Tactile Exploration}

With tactile exploration the goal is to build up an accurate model of the shape of an unknown object. One early paper by Goldberg and Bajcsy (1984) described a system that showed that very little information is necessary to reconstruct an unknown shape. With some parametrized shape models, a large variety of shapes can still be characterized. In (Fearing, 1990), for instance, results are given for recovering generalized cylinders. In (Chen et al., 1996) tactile data are fit to a general quadratic form. Finally, (Roberts, 1990) proposed a tactile exploration method for polyhedra.

Allen and Michelman (1990) presented methods for exploring shapes in three stages, from coarse to fine: grasping by containment, planar surface exploring and surface contour following. Montana (1988) describes a method to estimate curvature based on a number of probes. Montana also presents a control law for contour following. Charlebois et al. $(1996,1997)$ introduced two different tactile exploration methods: one uses Montana's contact equations and one fits a B-spline surface to the contact points and normals obtained by sliding multiple fingers along a surface.

Marigo et al. (1997) showed how to manipulate a known polyhedral part by rolling between the two palms of a paralleljaw gripper. Recently, (Bicchi et al., 1999) extended these results to tactile exploration of unknown objects with a paralleljaw gripper equipped with tactile sensors. A different ap- proach is taken by Kaneko and Tsuji (2000), who try to recover the shape by pulling a finger over the surface. This idea has also been explored by Russell (1992). In (Okamura and Cutkosky, 1999) the emphasis is on detecting fine surface features such as bumps and ridges.

Much of our work builds forth on (Erdmann, 1998b). Erdmann derives the shape of an unknown object with an unknown motion as a function of the sensor values. In our work we restrict the motion of the object: we assume quasistatic dynamics and we assume there is no friction. Only gravity and the contact forces are acting on the object. As a result we can recover the shape with fewer sensors. We can realize these assumptions by moving the palms slowly enough so that the object is always in a local potential energy minimum.

\subsection{Tactile Sensor Design}

Despite the large body of work in tactile sensing and haptics, making reliable and accurate tactile sensors has proven to be very hard. Many different designs have been proposed. For an overview of sensing technologies, see e.g. (Howe and Cutkosky, 1992). Fearing and Binford (1988) describe a cylindrical tactile sensor to determine the curvature of convex unknown shapes. In our own experiments we will rely on off-the-shelf components. The actual tactile sensors are touchpads as found on many notebooks. Most touchpads use capacitive technology, but the ones we are using are based on force-sensing resistors, which are less sensitive to electrostatic contamination.

\section{Quasistatic Shape Reconstruction}

In this section we will present a quasistatic method for reconstructing the shape of an unknown smooth convex object. The object is placed between the two palms, and we can vary the angles between the palms and the world frame. We say that the object is in force/torque balance, if and only if all forces and torques acting on the object add up to 0. Below, we will show that if we assume that the object is always in force/torque balance and if there is no friction between the object and the palms, then we can reconstruct the shape with two palms.

Figure 1(b) shows the two inputs and the two sensor outputs. The inputs are $\phi_{1}$, the angle between palm 1 and the $\mathrm{X}$-axis of the global frame, and $\phi_{2}$, the angle between palm 1 and 2. The tactile sensor elements return the contact points $s_{1}$ and $s_{2}$ on palm 1 and 2, respectively. Gravity acts in the negative $\mathrm{Y}$ direction. If the object is at rest, there is force/torque balance. In that case, since we assume there is no friction, the lines through the normal forces at the contact points and gravity acting on the center of mass intersect at a common point. In other words, the sensor values tell us where the $\mathrm{X}$-coordinate of the center of mass is in the global frame. Below we will show that this constraint on the position of the center of mass and the constraints induced by the sensor 


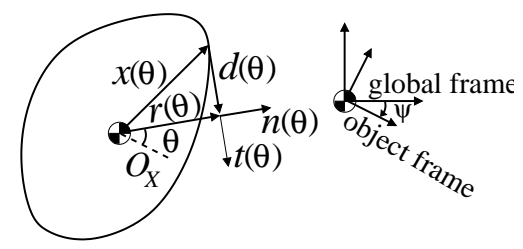

Figure 2: The contact support function $(r(\theta), d(\theta))$

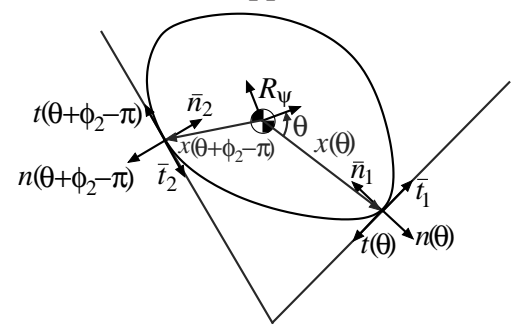

Figure 3: The different coordinate frames

values will allow us to derive an expression for the curvature at the contact points. However, this expression depends on the initial Y-coordinate of the center of mass. We can search for this value by minimizing the error between what the curvature expression predicts and what the sensor values tell us.

A useful tool for recovering the shape of the object will be the radius function (see e.g. (Santaló, 1976)). Figure 2 shows the basic idea. We assume that the object is smooth and convex. We also assume that the origin of the object frame is at the center of mass. For every angle $\theta$ we can construct a line $\ell(\theta)$ tangent to the object. This line makes contact with the object at $\mathbf{x}(\theta)$. So $\mathbf{x}(\theta)$ describes the shape of the object, with $\theta$ varying over $[0,2 \pi)$. Let $\mathbf{n}(\theta)$ be the outward pointing normal and $\mathbf{t}(\theta)$ be the tangent such that $[\mathbf{t}, \mathbf{n}]$ constitutes a right-handed frame. Then $\mathbf{n}(\theta)=(\cos \theta, \sin \theta)^{T}$ and $\mathbf{t}(\theta)=$ $(\sin \theta,-\cos \theta)^{T}$. Similarly, we can define frames at the contact points with respect to the palms:

$$
\begin{aligned}
& \overline{\mathbf{n}}_{1}=\left(-\sin \phi_{1}, \cos \phi_{1}\right)^{T} \text { and } \quad \overline{\mathbf{n}}_{2}=\left(\sin \phi_{12},-\cos \phi_{12}\right)^{T} \\
& \overline{\mathbf{t}}_{1}=\left(\cos \phi_{1}, \sin \phi_{1}\right)^{T} \text { and } \overline{\mathbf{t}}_{2}=-\left(\cos \phi_{12}, \sin \phi_{12}\right)^{T}
\end{aligned}
$$

Here, $\phi_{12}=\phi_{1}+\phi_{2}$. Let $\psi$ be the angle between the object frame and the global frame, such that a rotation matrix $\mathbf{R}(\psi)$ maps a point from the object frame to the global frame:

$$
\mathbf{R}(\psi)=\left(\begin{array}{cc}
\cos \psi & -\sin \psi \\
\sin \psi & \cos \psi
\end{array}\right)
$$

The object and palm frames are then related in the following way:

$$
\begin{aligned}
& \left(\begin{array}{ll}
\overline{\mathbf{n}}_{1} & \overline{\mathbf{t}}_{1}
\end{array}\right)=-\mathbf{R}(\psi)(\mathbf{n}(\theta) \quad \mathbf{t}(\theta)) \\
& \left(\begin{array}{ll}
\overline{\mathbf{n}}_{2} & \left.\overline{\mathbf{t}}_{2}\right)=-\mathbf{R}(\psi)\left(\mathbf{n}\left(\theta+\phi_{2}-\pi\right)\right. \\
\left.\mathbf{t}\left(\theta+\phi_{2}-\pi\right)\right)
\end{array}\right.
\end{aligned}
$$

The different frames are shown in figure 3 . From these relationships it follows that

$$
\theta=\phi_{1}-\psi-\frac{\pi}{2}
$$

We will use "." to represent differentiation with respect to time $t$ and " "to represent differentiation with respect to a function's parameter. So, for instance, $\dot{\mathbf{x}}(\theta)=\mathbf{x}^{\prime}(\theta) \dot{\theta}$.

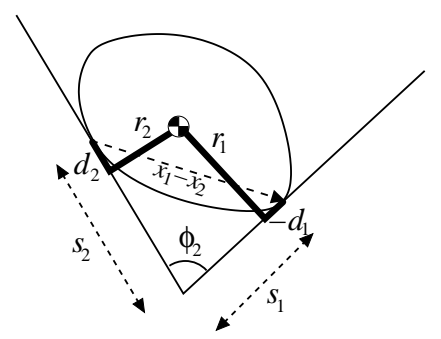

Figure 4: The dependencies between sensor values, the support function and the angle between the palms

From the Frenet formulas it follows that the parameterization velocity $v(\theta)=\left\|\mathbf{x}^{\prime}(\theta)\right\|$ is the radius of curvature of the shape at the point $\mathbf{x}(\theta)$. We can write $v(\theta)$ as $-\mathbf{x}^{\prime}(\theta) \cdot \mathbf{t}(\theta)$ and $\mathbf{x}^{\prime}(\theta)$ as $-v(\theta) \mathbf{t}(\theta)$.

We now define $r(\theta)$ to be the distance between $\ell(\theta)$ and the object origin:

$$
r(\theta)=\mathbf{x}(\theta) \cdot \mathbf{n}(\theta)
$$

This function is called a radius function or support function. For our shape recovery analysis it will be useful to define another function, $d(\theta)$, to be the signed distance of the contact point $x(\theta)$ to the foot of the supporting line $\ell(\theta)$ :

$$
d(\theta)=\mathbf{x}(\theta) \cdot \mathbf{t}(\theta)
$$

We will refer to the pair $(r(\theta), d(\theta))$ as a contact support function. The goal is now to derive a solution for $\mathbf{x}(\theta)$ as we change the palm angles $\phi_{1}$ and $\phi_{2}$. Below we drop the function arguments where it doesn't lead to confusion, and instead use subscripts ' 1 ' and ' 2 ' to denote the contact point on palm 1 and 2. So we will write e.g. $r_{2} \mathbf{n}_{2}$ for $r\left(\theta+\phi_{2}-\right.$ $\pi) \mathbf{n}\left(\theta+\phi_{2}-\pi\right)$. Note that $r^{\prime}(\theta)=-d(\theta)$, so it is sufficient to reconstruct the radius function. If the object is in two-point contact, $d(\theta)$ is redundant in another way as well. We can write the two-point contact constraint in terms of the contact support function:

$$
\left(s_{1}+d_{1}\right) \overline{\mathbf{t}}_{1}+r_{1} \overline{\mathbf{n}}_{1}=\left(-s_{2}+d_{2}\right) \overline{\mathbf{t}}_{2}+r_{2} \overline{\mathbf{n}}_{2}
$$

Solving this constraint for $d_{1}$ and $d_{2}$ we get:

$$
d_{1}=\frac{r_{1} \cos \phi_{2}+r_{2}}{\sin \phi_{2}}-s_{1} \quad \text { and } \quad d_{2}=-\frac{r_{2} \cos \phi_{2}+r_{1}}{\sin \phi_{2}}+s_{2}
$$

See also figure 4. So a solution for $r(\theta)$ can be used in two ways to arrive at a solution for $d(\theta)$ : (1) using the property $d(\theta)=-r^{\prime}(\theta)$ of the radius function, or (2) using the expressions above.

One final bit of notation we need is a generalization of the contact support function, which we will define as a projection of the vector between the two contact points. We define the generalized contact support function relative to contact point 1 as:

$$
\begin{aligned}
& \tilde{r}_{1}(\theta)=\left(\mathbf{x}(\theta)-\mathbf{x}\left(\theta+\phi_{2}-\pi\right)\right) \cdot \mathbf{n}(\theta) \\
& \tilde{d}_{1}(\theta)=\left(\mathbf{x}(\theta)-\mathbf{x}\left(\theta+\phi_{2}-\pi\right)\right) \cdot \mathbf{t}(\theta)
\end{aligned}
$$

Similarly, we can define the generalized contact support function relative to contact point 2 as:

$$
\begin{aligned}
& \tilde{r}_{2}(\theta)=\left(\mathbf{x}(\theta)-\mathbf{x}\left(\theta+\phi_{2}-\pi\right)\right) \cdot \mathbf{n}\left(\theta+\phi_{2}-\pi\right) \\
& \tilde{d}_{2}(\theta)=\left(\mathbf{x}(\theta)-\mathbf{x}\left(\theta+\phi_{2}-\pi\right)\right) \cdot \mathbf{t}\left(\theta+\phi_{2}-\pi\right)
\end{aligned}
$$






Figure 5: The generalized contact support functions.

The generalized contact support functions have the property that they can be expressed directly in terms of the palms angles and sensor values (assuming the object is in two-point contact):

$$
\begin{aligned}
& \tilde{r}_{1}=s_{2} \sin \phi_{2} \\
& \tilde{d}_{1}=s_{2} \cos \phi_{2}-s_{1}
\end{aligned} \quad \text { and } \quad \begin{aligned}
& \tilde{r}_{2}=-s_{1} \sin \phi_{2} \\
& \tilde{d}_{2}=s_{1} \cos \phi_{2}-s_{2}
\end{aligned}
$$

These equalities can be obtained by inspection from figures 1(b) and 5. By differentiating the generalized contact support functions with respect to time we can obtain the following two expressions for the radii of curvature:

$$
v_{1}=-\frac{\dot{\tilde{r}}_{2}+\left(\dot{\theta}+\dot{\phi}_{2}\right) \tilde{d}_{2}}{\dot{\theta} \sin \phi_{2}} \quad \text { and } \quad v_{2}=-\frac{\dot{\tilde{r}}_{1}+\dot{\theta} \tilde{d}_{1}}{\left(\theta+\dot{\phi}_{2}\right) \sin \phi_{2}}
$$

So we can observe the curvature at the contact points if we can derive an expression for $\dot{\theta}$ as a function of sensor values and palm angles. Equivalently, we can derive an expression for $\dot{\psi}$, since it follows from equation 1 that $\dot{\theta}=\dot{\phi}_{1}-\dot{\psi}$. It can be shown that the lines through the normals intersect at $s_{1} \overline{\mathbf{t}}_{1}-\tilde{d}_{2} \overline{\mathbf{n}}_{1} / \sin \phi_{2}$. Therefore, if the object is in force/torque balance, the distance between the center of mass and contact point 1 along the $\mathrm{X}$-axis can be written as

$$
\left(\mathbf{R x}_{1}\right) \cdot\left(\begin{array}{l}
1 \\
0
\end{array}\right)=-\tilde{d}_{2} \frac{\sin \phi_{1}}{\sin \phi_{2}}
$$

The left-hand side of this equation can be rewritten as

$$
\begin{aligned}
\left(\mathbf{R} \mathbf{x}_{1}\right) \cdot\left(\begin{array}{l}
1 \\
0
\end{array}\right) & =\left(\mathbf{R}\left(r_{1} \mathbf{n}_{1}+d_{1} \mathbf{t}_{1}\right)\right) \cdot\left(\begin{array}{l}
1 \\
0
\end{array}\right) \\
& =r_{1} \sin \phi_{1}-d_{1} \cos \phi_{1}
\end{aligned}
$$

Differentiating expression 12 we get:

$$
\begin{aligned}
\frac{d}{d t} & \left(r_{1} \sin \phi_{1}-d_{1} \cos \phi_{1}\right) \\
= & \left(\dot{r}_{1}+d_{1} \dot{\phi}_{1}\right) \sin \phi_{1}+\left(r_{1} \dot{\phi}_{1}-\dot{d}_{1}\right) \cos \phi_{1} \\
= & d_{1}\left(\dot{\phi}_{1}-\dot{\theta}\right) \sin \phi_{1}+r_{1}\left(\dot{\phi}_{1}-\dot{\theta}\right) \cos \phi_{1} \\
& -\frac{\dot{\tilde{r}}_{2}+\left(\dot{\theta}+\dot{\phi}_{2}\right) \tilde{d}_{2}}{\sin \phi_{2}} \cos \phi_{1} \\
= & \dot{\psi}\left(d_{1} \sin \phi_{1}+r_{1} \cos \phi_{1}+\tilde{d}_{2} \frac{\cos \phi_{1}}{\sin \phi_{2}}\right) \\
& -\frac{\dot{\tilde{r}}_{2}+\left(\dot{\phi}_{1}+\dot{\phi}_{2}\right) \tilde{d}_{2}}{\sin \phi_{2}} \cos \phi_{1}
\end{aligned}
$$

The step in equation 15 follows from properties of the contact support function: $r^{\prime}(\theta)=-d(\theta)$ and $d^{\prime}(\theta)=r(\theta)-v(\theta)$. The derivative of the right-hand side of equation 10 can be written as

$$
\left(-\dot{\tilde{d}}_{2} \sin \phi_{1}-\tilde{d}_{2} \dot{\phi}_{1} \cos \phi_{1}+\tilde{d}_{2} \dot{\phi}_{2} \sin \phi_{1} \cot \phi_{2}\right) / \sin \phi_{2}
$$

Equating expressions 16 and 17, substituting expression 3 for $d_{1}$, and solving for $\dot{\psi}$ we arrive at the following expression for $\dot{\psi}$ :

$$
\dot{\psi}=\frac{\dot{\tilde{r}}_{2} \cos \phi_{1}-\dot{\tilde{d}}_{2} \sin \phi_{1}+\tilde{d}_{2} \dot{\phi}_{2} \frac{\sin \phi_{12}}{\sin \phi_{2}}}{r_{1} \sin \phi_{12}+\left(r_{2}+\tilde{r}_{2}\right) \sin \phi_{1}+\tilde{d}_{2} \cos \phi_{1}}
$$

This expression for $\dot{\psi}$ depends on the control inputs, the sensor values, their derivatives and the values of radius function at the contact points. The system of differential equations describing the (sensed) shape and motion can be summarized as follows:

$$
\begin{aligned}
& \dot{r}_{1}=-d_{1}\left(\dot{\phi}_{1}-\dot{\psi}\right) \\
& \dot{r}_{2}=-d_{2}\left(\dot{\phi}_{12}-\dot{\psi}\right) \\
& \dot{\psi}=\frac{\dot{\tilde{r}}_{2} \cos \phi_{1}-\dot{\tilde{d}}_{2} \sin \phi_{1}+\tilde{d}_{2} \dot{\phi}_{2} \frac{\sin \phi_{12}}{\sin \phi_{2}}}{r_{1} \sin \phi_{12}+\left(r_{2}+\tilde{r}_{2}\right) \sin \phi_{1}+\tilde{d}_{2} \cos \phi_{1}}
\end{aligned}
$$

In our current implementation we use a fourth-order AdamsBashforth-Moulton predictor-corrector method to integrate this system of differential equations. This high-order method tends to filter out most of the noise. More research is necessary to determine the sensitivity of the system to noise.

The differential equations describe the local shape, but they do not directly address the question whether it is always possible to reconstruct the global shape. In (Moll and Erdmann, 2001) we prove that the shape of a random smooth convex object is, in fact, globally observable. The stable poses induce a two-dimensional subset of the $\left(\phi_{1}, \phi_{2}, \psi\right)$ configuration space. We prove global observability by analyzing the boundary conditions of this subset.

So far we have assumed that we have sensor data that is continuous and without any error. In practice sensors will be discrete, both in time and space, and there will also be errors. We would like to recover the shape of an unknown object in such a setting as well. Locally we can observe two errors:

1. The difference between the predicted and actual position of the X-coordinate of the center of mass. Here, 'predicted' means: found by integrating the differential equations 19- 21.

2. The difference between the predicted and actual contact point vector.

By minimizing the sum of all locally observable errors we search for the initial conditions.

\section{Simulation Results}

Figure 6 shows an example of the shape reconstruction process. The results are based on numerical simulation. 270 measurements were used to reconstruct the shape. The motion of the palms is open-loop. Initially palm 1 and palm 2 are nearly horizontal; the object is squeezed (but without friction!) between the palms. The motion of the palms can roughly be described as sequence of squeeze-and-rotate motions and motions where one of the palms stays put and the other palm opens up. Notice how in the penultimate frame 

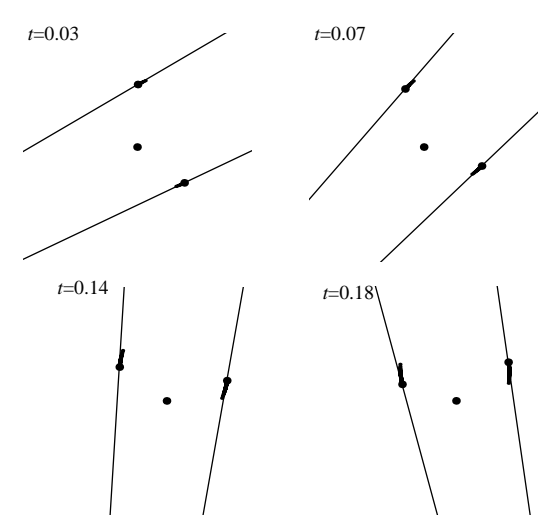

$t=0.26$
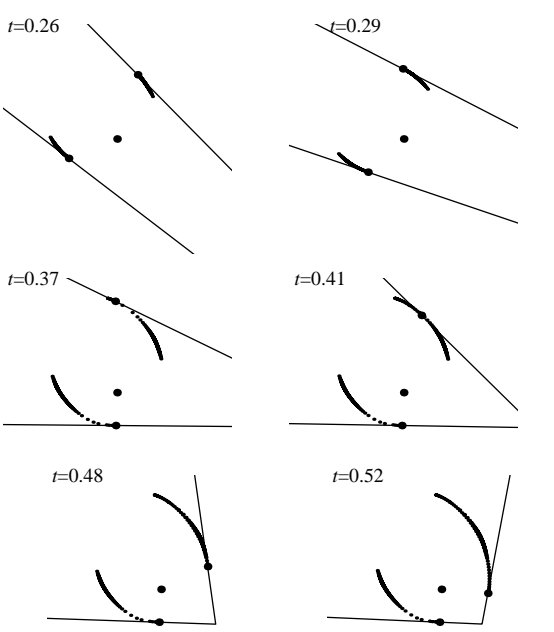

$t=0.59$

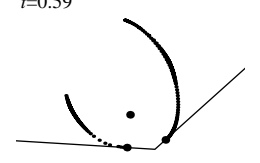

$t=0.70$
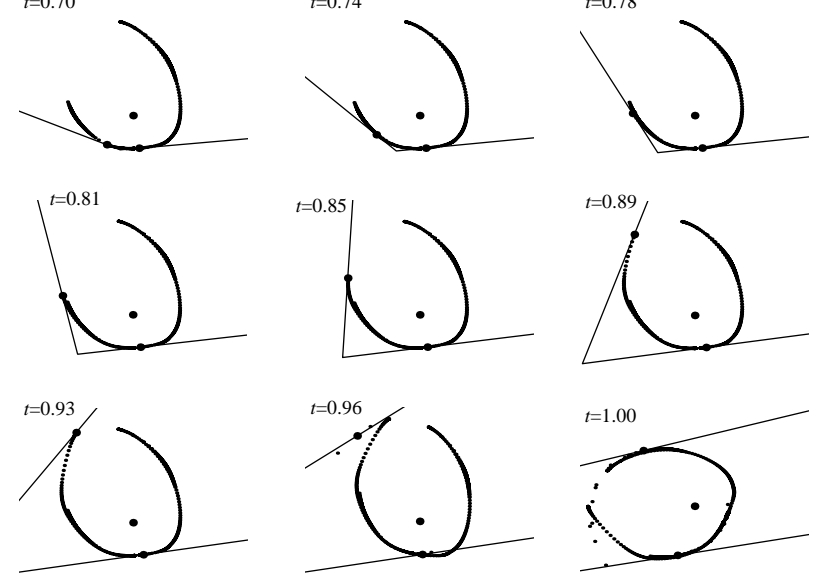

Figure 6: The frames show the reconstructed shape after 10, $20, \ldots, 270$ measurements. The three large dots indicate the center of mass and the contact points at each time, the smaller dots show the part of the shape that has been reconstructed at that time.
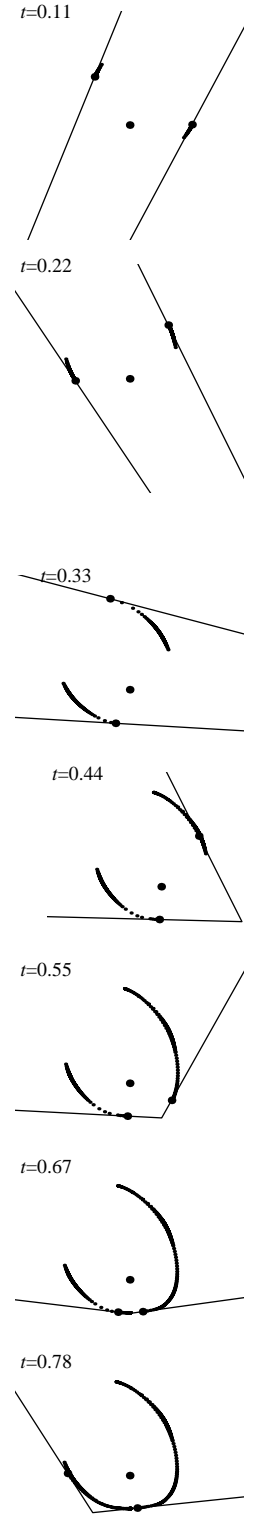

(a) The actual shape and the observed shape.

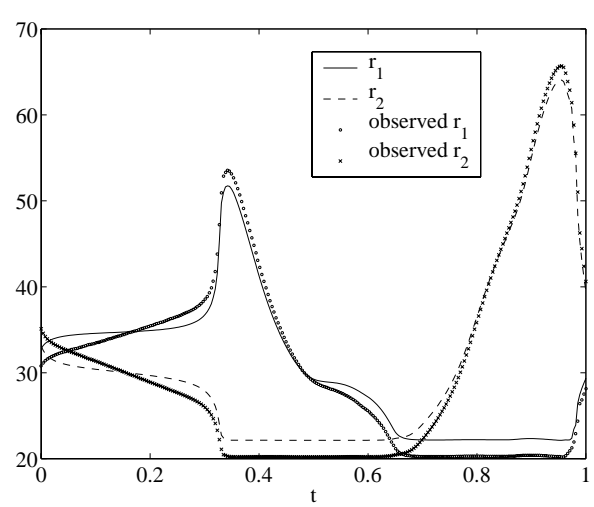

(b) The actual and observed values of the radius function.

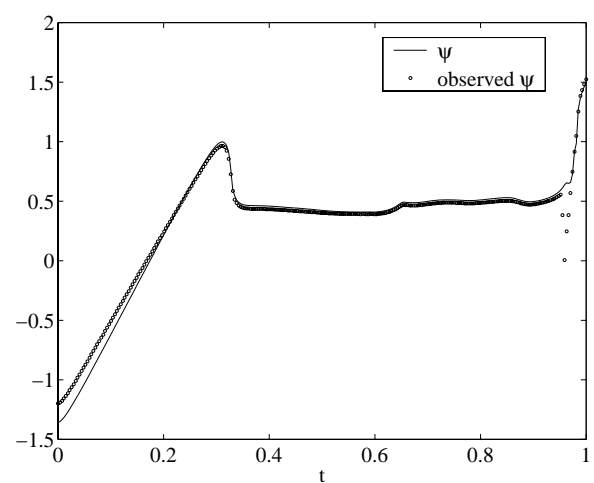

(c) The actual and observed orientation of the object.

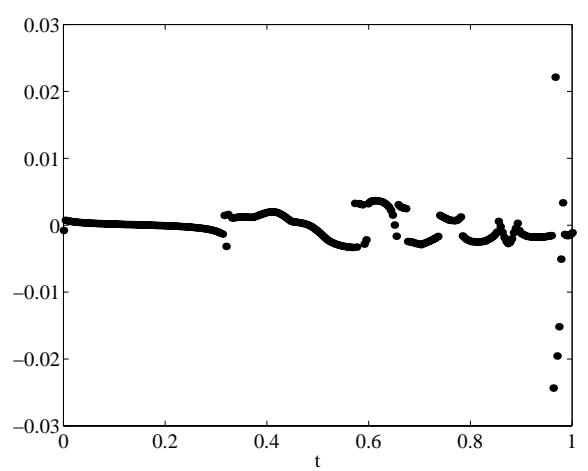

(d) The error in the norm of the contact point vector.

Figure 7: The differences between the actual and observed shape. 


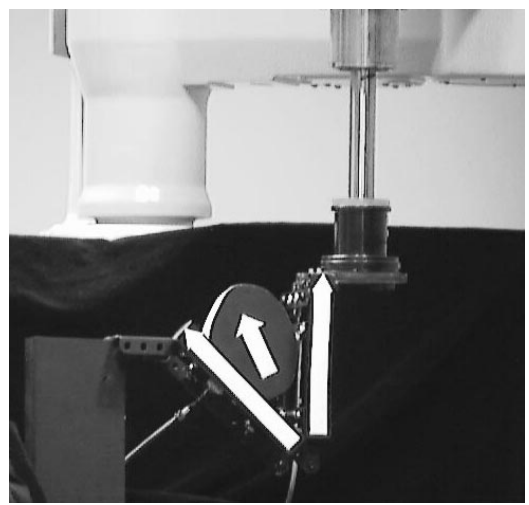

(a) Experimental setup

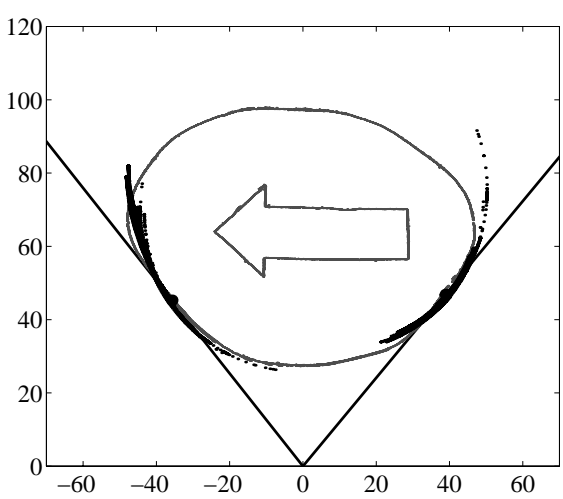

(b) Partially reconstructed shape

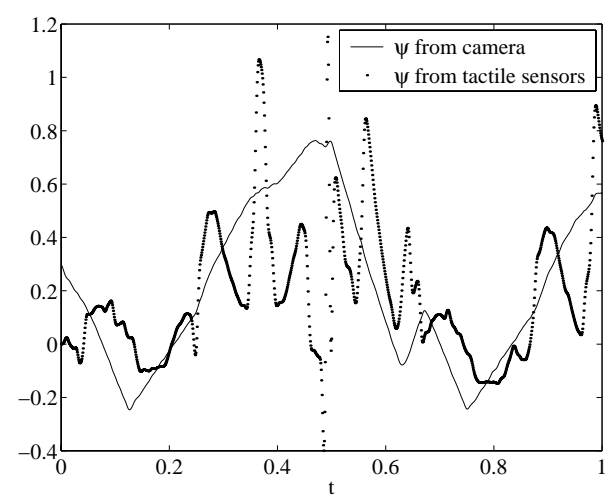

(c) Orientation measured by the vision system and the observed orientation

Figure 8: Experimental Results

the simulator misgauges the shape, but has recovered in the last frame.

In figure 7 the differences are shown between the reconstructed and actual shape and motion of the object. We don't know the initial orientation, but in some sense the choice of initial orientation is arbitrary. We use SVD to align the orientation of the actual and observed shape (Golub and Loan, 1996, p. 601). The error in the norm of the contact point vector is very small, but doesn't appear to be completely random, suggesting that there is still room for improvement in the integration step.

\section{Experimental Results}

Our experimental setup is shown in figure 8(a). An Adept SCARA robot arm holds the endpoint of palm 1. The endpoint of palm 2 is attached to a fixed base. On each palm there is one touchpad from Interlink Electronics. The touchpads use so-called force sensing resistors to measure the location and the applied pressure at the contact point. If there is more than one contact point, the pad returns the centroid. The physical pads have a resolution of 1000 counts per inch (CPI) in the $\mathrm{X}$ and $\mathrm{Y}$ direction, but the firmware limits the resolution to 200 CPI. The pads can report 128 pressure levels. The pads measure $55.5 \times 39.5 \mathrm{~mm}^{2}$. Sensor data can be read out at a rate of approximately $100 \mathrm{~Hz}$ through a RS232 serial port connection. The white arrows on the object and the palms are tracked by an Adept vision system in order to establish 'ground truth', which can be compared with the shape and motion inferred from the tactile data.

Figures 8(b) and 8(c) show the reconstructed shape and motion, respectively. The observed motion is far from perfect, but the observed shape comes close to the actual shape. This seems to suggest that the system of differential equations 19-21 is fairly stable in this case, i.e., the errors in the motion did not cause the radius function to shoot off to infinity. The palms made back-and-forth motions in order to cover the shape several times. This means that we can prune those parts of the reconstructed shape that have been touched only once. For instance, in figure 8(b) we can eliminate the sparse point distribution in the top right and bottom middle.

\section{Discussion}

In this paper we have shown how to reconstruct the shape of an unknown smooth convex planar shape using two tactile sensors. We derived expressions for the curvature at the contact points and the rotational speed of the object. The simulation results showed that our approach works well, but some work remains to be done on finding the initial conditions. Also, it is possible that the object undergoes a discrete change in orientation (when a local minimum of the potential energy function becomes a local maximum). In that case we may need to restart the integration and search again for the initial conditions at that point. We will then also need to find the most likely arrangement of the different shape segments.

In (Moll and Erdmann, 2001) we addressed the dynamic case, where force/torque balance is no longer assumed. We established that it is possible to reconstruct the shape in this case as well as long as at least one of the palms is moving. By moving the palms slowly enough, we can approximate the quasistatic case, which seems to suggest that we may be able to achieve global observability in the dynamic case as well. Further research is needed to confirm this intuition. If both palms are motionless, the shape is observable if the robot can measure the contact forces.

In future work we hope to extend our analytic results in the following ways. Firstly, we are planning to extend our model of the dynamics to include friction. We hope to be able to reconstruct the value of the friction coefficient using a nonlinear observer (Isidori, 1995). Secondly, we will analyze the three-dimensional case. In 3D we cannot expect to reconstruct the entire shape, since the contact point trace out only curves on the surface of the object. Nevertheless, by constructing a sufficiently fine mesh with these curves, we can come up with a good approximation. The quasistatic approach will most likely not work in $3 \mathrm{D}$, because in $3 \mathrm{D}$ the rotation velocity has three degrees of freedom and force/torque balance only gives us two constraints. 


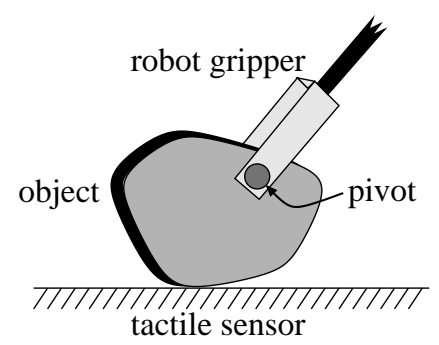

Figure 9: Dragging an object over a tactile sensor with a pivoting grasp

Figure 9 shows an entirely different sensing strategy. The object is grasped by a robot arm using a pivoting gripper (Rao et al., 1994, 1995). With such a gripper the object is free to rotate around the line through the grasp contact points. The sensing strategy consists of dragging or pushing the object over a surface coated with tactile sensors. We think it would be interesting to determine whether this system is observable as well.

\section{Acknowledgments}

The authors wish to thank Yan-Bin Jia, Matt Mason, Al Rizzi, Garth Zeglin and Devin Balkcom for helpful discussions.

\section{References}

Akella, S. and Mason, M. T. (1998). Parts orienting with partial sensor information. In Proc. 1998 IEEE Intl. Conf. on Robotics and Automation, pages 557-564, Leuven, Belgium.

Allen, P. K. and Michelman, P. (1990). Acquisition and interpretation of 3-D sensor data from touch. IEEE Trans. on Robotics and Automation, 6(4):397-404.

Arkin, E., Held, M., Mitchell, J., and Skiena, S. (1998). Recognizing polygonal parts from width measurements. Computational Geometry: Theory and Applications, 9(4):237-246.

Bicchi, A., Marigo, A., and Prattichizzo, D. (1999). Dexterity through rolling: Manipulation of unknown objects. In Proc. 1999 IEEE Intl. Conf. on Robotics and Automation, pages 1583-1588, Detroit, Michigan.

Boissonnat, J. D. and Yvinec, M. (1992). Probing a scene of nonconvex polyhedra. Algorithmica, 8:321-342.

Cai, C. S. and Roth, B. (1986). On the planar motion of rigid bodies with point contact. Mechanism and Machine Theory, 21(6):453466.

Cai, C. S. and Roth, B. (1987). On the spatial motion of a rigid body with point contact. In Proc. 1987 IEEE Intl. Conf. on Robotics and Automation, pages 686-695.

Charlebois, M., Gupta, K., and Payandeh, S. (1996). Curvature based shape estimation using tactile sensing. In Proc. 1996 IEEE Intl. Conf. on Robotics and Automation, pages 3502-3507.

Charlebois, M., Gupta, K., and Payandeh, S. (1997). Shape description of general, curved surfaces using tactile sensing and surface normal information. In Proc. 1997 IEEE Intl. Conf. on Robotics and Automation, pages 2819-2824.

Chen, N., Rink, R., and Zhang, H. (1996). Local object shape from tactile sensing. In Proc. 1996 IEEE Intl. Conf. on Robotics and Automation, pages 3496-3501.
Cole, R. and Yap, C. K. (1987). Shape from probing. Journal of Algorithms, 8(1):19-38.

Dobkin, D., Edelsbrunner, H., and Yap, C. K. (1986). Probing convex polytopes. In Proceedings of the Eighteenth Annual ACM Symposium on Theory of Computing, pages 424-432, Berkeley, California.

Erdmann, M. A. (1998a). An exploration of nonprehensile twopalm manipulation: Planning and execution. Intl. J. of Robotics Research, 17(5).

Erdmann, M. A. (1998b). Shape recovery from passive locally dense tactile data. In Workshop on the Algorithmic Foundations of Robotics.

Erdmann, M. A. and Mason, M. T. (1988). An exploration of sensorless manipulation. IEEE J. of Robotics and Automation, 4(4):369-379.

Erdmann, M. A., Mason, M. T., and Vaněček, Jr., G. (1993). Mechanical parts orienting: The case of a polyhedron on a table. Algorithmica, 10:226-247.

Fearing, R. S. (1990). Tactile sensing for shape interpretation. In Venkataraman, S. T. and Iberall, T., editors, Dexterous Robot Hands, chapter 10, pages 209-238. Springer Verlag, Berlin; Heidelberg; New York.

Fearing, R. S. and Binford, T. O. (1988). Using a cylindrical tactile sensor for determining curvature. In Proc. 1988 IEEE Intl. Conf. on Robotics and Automation, pages 765-771.

Goldberg, K. Y. and Bajcsy, R. (1984). Active touch and robot perception. Cognition and Brain Theory, 7(2):199-214.

Golub, G. H. and Loan, C. F. V. (1996). Matrix Computations. Johns Hopkins University Press, third edition.

Howe, R. D. and Cutkosky, M. R. (1992). Touch sensing for robotic manipulation and recognition. In Khatib, O. and Lozano-Pérez, J. J. C. T., editors, The Robotics Review 2. MIT Press, Cambridge, MA.

Isidori, A. (1995). Nonlinear Control Systems. Springer Verlag, Berlin; Heidelberg; New York, third edition.

Jia, Y.-B. (2000). Grasping curved objects through rolling. In Proc. 2000 IEEE Intl. Conf. on Robotics and Automation, pages 377382, San Francisco, California.

Jia, Y.-B. and Erdmann, M. (1996). Geometric sensing of known planar shapes. Intl. J. of Robotics Research, 15(4):365-392.

Jia, Y.-B. and Erdmann, M. A. (1999). Pose and motion from contact. Intl. J. of Robotics Research, 18(5).

Kaneko, M. and Tsuji, T. (2000). Pulling motion based tactile sensing. In Workshop on the Algorithmic Foundations of Robotics, Hanover, New Hampshire.

Kao, I. and Cutkosky, M. R. (1992). Quasistatic manipulation with compliance and sliding. Intl. J. of Robotics Research, 11(1):2040.

Keren, D., Rivlin, E., Shimsoni, I., and Weiss, I. (1998). Recognizing surfaces using curve invariants and differential properties of curves and surfaces. In Proc. 1998 IEEE Intl. Conf. on Robotics and Automation, pages 3375-3381, Leuven, Belgium.

Klatzky, R. L., Lederman, S. J., and Metzger, V. A. (1985). Identifying objects by touch: An "expert system". Perception and Psychophysics, 37:299-302.

Li, S.-Y. R. (1988). Reconstruction of polygons from projections. Information Processing Letters, 28:235-240.

Lindenbaum, M. and Bruckstein, A. (1991). Parallel strategies for geometric probing. In Proc. 1991 IEEE Intl. Conf. on Robotics 
and Automation, pages 392-397.

Lindenbaum, M. and Bruckstein, A. M. (1994). Blind approximation of planar convex sets. IEEE Trans. on Robotics and Automation, 10(4):517-529.

Lynch, K. M. (1997). Nonprehensile Robotic Manipulation: Controllability and Planning. $\mathrm{PhD}$ thesis, Robotics Institute, Carnegie Mellon University, Pittsburgh, PA.

Lynch, K. M., Shiroma, N., Arai, H., and Tanie, K. (1998). The roles of shape and motion in dynamic manipulation: The butterfly example. In Proc. 1998 IEEE Intl. Conf. on Robotics and Automation.

Marigo, A., Chitour, Y., and Bicchi, A. (1997). Manipulation of polyhedral parts by rolling. In Proc. 1997 IEEE Intl. Conf. on Robotics and Automation, pages 2992-2997.

Mason, M. T. (1982). Manipulator Grasping and Pushing Operations. PhD thesis, AI-TR-690, Artificial Intelligence Laboratory, MIT.

Mason, M. T. (1985). The mechanics of manipulation. In Proc. 1985 IEEE Intl. Conf. on Robotics and Automation, pages 544 548 , St. Louis.

Moll, M. and Erdmann, M. A. (2001). Shape reconstruction in a planar dynamic environment. Technical Report CMU-CS-01107, Dept. of Computer Science, Carnegie Mellon University. http://www.cs.cmu.edu/ $\sim$ mmoll/publications.

Montana, D. J. (1988). The kinematics of contact and grasp. Intl. J. of Robotics Research, 7(3):17-32.

Montana, D. J. (1995). The kinematics of multi-fingered manipulation. IEEE Trans. on Robotics and Automation, 11(4):491-503.

Nagata, K., Keino, T., and Omata, T. (1993). Acquisition of an object model by manipulation with a multifingered hand. In Proc. 1993 IEEE/RSJ Intl. Conf. on Intelligent Robots and Systems, volume 3, pages 1045-1051, Osaka, Japan.

Okamura, A. M. and Cutkosky, M. R. (1999). Haptic exploration of fine surface features. In Proc. 1999 IEEE Intl. Conf. on Robotics and Automation, pages 2930-2936, Detroit, Michigan.

Paljug, E., Yun, X., and Kumar, V. (1994). Control of rolling contacts in multi-arm manipulation. IEEE Trans. on Robotics and Automation, 10(4):441-452.

Peshkin, M. A. and Sanderson, A. C. (1988). Planning robotic manipulation strategies for sliding objects. IEEE J. of Robotics and Automation, 4(5).

Rao, A., Kriegman, D., and Goldberg, K. (1994). Complete algorithms for feeding polyhedral parts using pivot grasps. Technical Report UU-CS-1994-49, Utrecht University. ftp://ftp.cs.ruu.nl/ pub/RUU/CS/techreps/CS-1994/1994-49.ps.gz.

Rao, A., Kriegman, D., and Goldberg, K. (1995). Complete algorithms for reorienting polyhedral parts using a pivoting gripper. In Proc. 1995 IEEE Intl. Conf. on Robotics and Automation.

Rao, A. S. and Goldberg, K. Y. (1994). Shape from diameter: Recognizing polygonal parts with a parallel-jaw gripper. Intl. J. of Robotics Research, 13(1):16-37.

Rizzi, A. A. and Koditschek, D. E. (1993). Further progress in robot juggling: The spatial two juggle. In Proc. 1993 IEEE Intl. Conf. on Robotics and Automation, volume 3, pages 919-924, Atlanta, Georgia.

Roberts, K. S. (1990). Robot active touch exploration: Constraints and strategies. In Proc. 1990 IEEE Intl. Conf. on Robotics and Automation, pages 980-985.

Russell, R. A. (1992). Using tactile whiskers to measure surface contours. In Proc. 1992 IEEE Intl. Conf. on Robotics and Automation, volume 2, pages 1295-1299, Nice, France.

Salisbury, K. (1987). Whole arm manipulation. In Proc. Fourth Intl. Symp. on Robotics Research, pages 183-189, Santa Cruz, California.

Santaló, L. A. (1976). Integral Geometry and Geometric Probability, volume 1 of Encyclopedia of Mathematics and its Applications. Addison-Wesley, Reading, MA.

Schneiter, J. L. and Sheridan, T. B. (1990). An automated tactile sensing strategy for planar object recognition and localization. IEEE Trans. on Pattern Analysis and Machine Intelligence, 12(8):775-786.

Siegel, D. M. (1991). Finding the pose of an object in a hand. In Proc. 1991 IEEE Intl. Conf. on Robotics and Automation, pages 406-411.

Skiena, S. S. (1989). Problems in geometric probing. Algorithmica, 4(4):599-605.

Sudsang, A., Ponce, J., and Srinivasa, N. (2000). In-hand manipulation: Geometry and algorithms. Algorithmica, 26(4):466-493.

Teichmann, M. and Mishra, B. (2000). Reactive robotics I: Reactive grasping with a modified gripper and multi-fingered hands. Intl. J. of Robotics Research, 19(7):697-708.

Trinkle, J. C., Abel, J. M., and Paul, R. P. (1988). An investigation of frictionless enveloping grasping in the plane. Intl. J. of Robotics Research, 7(3):33-51.

Trinkle, J. C. and Hunter, J. J. (1991). A framework for planning dexterous manipulation. In Proc. 1991 IEEE Intl. Conf. on Robotics and Automation, pages 1245-1251.

Trinkle, J. C. and Paul, R. P. (1990). Planning for dexterous manipulation with sliding contacts. Intl. J. of Robotics Research, 9(3):24-48.

Trinkle, J. C., Ram, R. C., Farahat, A. O., and Stiller, P. F. (1993). Dexterous manipulation planning and execution of an enveloped slippery workpiece. In Proc. 1993 IEEE Intl. Conf. on Robotics and Automation, pages 442-448.

Whitcomb, L. L., Rizzi, A. A., and Koditschek, D. E. (1993). Comparative experiments with a new adaptive controller for robot arms. IEEE Trans. on Robotics and Automation, 9(1):59-70.

Wiegley, J., Goldberg, K., Peshkin, M., and Brokowski, M. (1996). A complete algorithm for designing passive fences to orient parts. In Proc. 1996 IEEE Intl. Conf. on Robotics and Automation.

Yoshikawa, T., Yokokohji, Y., and Nagayama, A. (1993). Object handling by three-fingered hands using slip motion. In Proc. 1993 IEEE/RSJ Intl. Conf. on Intelligent Robots and Systems, pages 99-105, Yokohama, Japan.

Zumel, N. B. (1997). A Nonprehensile Method for Reliable Parts Orienting. $\mathrm{PhD}$ thesis, Robotics Institute, Carnegie Mellon University, Pittsburgh, PA. 